05

\title{
Согласование термодинамических свойств на линии плавления титана: проблемы, результаты
}

\author{
( Е.Ю. Кулямина, В.Ю. Зицерман, Л.Р. Фокин \\ Объединенный институт высоких температур РАН, \\ 125412 Москва, Россия \\ e-mail: kulyamina.elena@gmail.com
}

(Поступило в Редакцию 8 июня 2017 г.)

\begin{abstract}
Проведены анализ и согласование опытных данных о термодинамических свойствах на линии плавления титана в области от атмосферного давления до $90 \mathrm{GPa}$. Обсуждены проблемы: а) неполноты данных о плотности твердой $\beta$-фазы в области нормальной температуры плавления, б) вероятности образования на линии плавления тройной точки сосуществующих $\beta, \omega$ и жидкой фаз титана. Для оценки скачков объема при плавлении $3 d$-элементов периодической системы предложена и эффективно использована корреляция скачков объема $\Delta V_{m}$ и энтропии $\Delta S_{m}$ на линии плавления при атмосферном давлении.
\end{abstract}

DOI: 10.21883/JTF.2018.03.45594.2382

\section{Введение}

Через два года научная общественность будет отмечать 150 лет со времени первой публикации Д.И. Менделеева о периодической системе (ПС) элементов. За прошедшее время уровень знаний о свойствах элементов вырос многократно, возникло и развивается направление работ по корреляции свойств. И в то же время имеются разительные примеры неполноты данных о свойствах элементов не только в новых, но и в старых, традиционных областях исследования свойств. В круге наших научных интересов находятся вопросы согласования термодинамических свойств на линии плавления при температуре выше 1500-2000 К. Интерес к этой проблеме связан с тем, что: а) при столь высоких температурах традиционные методы экспериментальных исследований линий плавления веществ типа „поршень цилиндр“ не работают. Находят применение новые методы статических и динамических исследований, при этом достоверность соответствующих результатов нуждается в анализе и тестировании; б) при разработке широкодиапазонных параметрических уравнений состояния (УС) тугоплавких веществ [1] опытные данные о равновесии твердой и жидкой фаз являются естественным источником информации для определения параметров УС, с одной стороны, и используются для оценки качества самих УС, с другой; в) в последние десятилетия широкое развитие получили методы математического моделирования свойств конденсированных веществ на базе определяемых тем или иным способом, в прямой или обратной постановке задачи эффективных потенциалов межчастичных взаимодействий [2]. При этом опытные $T_{m}(p)$ данные, информация о производных $d T_{m} / d p$ на линии плавления используются для оценки качества результатов моделирования.

Наш опыт построения линий плавления тугоплавких металлов платиновой группы $\mathrm{Ru}\left(T_{m}^{0}=2607 \mathrm{~K}\right)$, Ir $\left(T_{m}^{0}=2707 \mathrm{~K}\right)$, Os $\left(T_{m}^{0}=3320 \mathrm{~K}\right)$ [3] показывает, что для этих элементов ПС отсутствуют не только надежные $T_{m}(p)$ данные при высоких давлениях, но и оценки производной на начальном участке линии плавления

$$
\left.\frac{d T_{m}}{d p}\right|_{p \rightarrow 0}=\frac{\Delta V_{m}}{\Delta S_{m}},
$$

где $\Delta V_{m}, \Delta S_{m}$ - скачки объема и энтропии в процессе плавления. Значение этой производной используется при анализе согласования опытных данных при высоком и атмосферном давлениях.

Основная цель работы заключается, с одной стороны, в том, чтобы провести анализ и согласование данных на линии плавления титана при высоких и низких давлениях и, с другой, показать неполноту и противоречивость информации на линии плавления элементов ПС, в частности титана, и необходимость в проведении исследований метрологического уровня факторов, влияющих на результаты измерений при высоких давлениях и температурах.

\section{Линия плавления титана}

Титан второй после скандия $3 d$-элемент ПС, $N=22$, молекулярный вес $M=47.88$, внешняя электронная оболочка атома $3 d^{2} 4 s^{2}$. При нагреве при атмосферном (нормальном) давлении гексагональная $\alpha$-фаза испытывает при $T=1156 \pm 3 \mathrm{~K}$ полиморфный $\alpha-\beta$-переход, который происходит с уменьшением объема $\delta V=$ $=-0.12 \pm 0.02 \%$. Новая $\beta$-фаза имеет ОЦК структуру, ее температура плавления $T_{m}^{0}=1944 \pm 3 \mathrm{~K}$ [4]. В справочниках по фазовым диаграммам элементов ПС $[5,6]$ информация о линии плавления Ті отсутствует, и только в новом издании [7] появляется сообщение о первом исследовании плавления $\beta$-фазы под давлением в интервале $15-80 \mathrm{GPa}$.

Еррандонеа и совторы [8] опубликовали результаты измерений температуры плавления $\mathrm{W}, \mathrm{Ta}, \mathrm{Mo}, \mathrm{V}$, Ti 
в интервале давлений $15-90 \mathrm{GPa}$, используя метод алмазных наковален (DAC-метод) с лазерным нагревом образца и определением момента плавления визуально или по термограмме процесса нагрева. Температура определялась с помощью многолучевого пирометра с использованием уравнения Планка для перехода от интенсивности излучения к термодинамической шкале температур. При этом проблема определения степени черноты образца на разных длинах волн, потери излучения по оптическому тракту в этой работе не обсуждаются, хотя оценки неопределенности температуры за счет этих факторов в подобных опытах могут достигать $10-15 \%$, т.е. в рассматриваемой области высоких температур величины 200-300 K [9]. Метод алмазных наковален является развитием метода измерения равновесия твердой и жидкой фаз при высоких давлениях с применением омического нагрева металла и использованием прозрачных в оптическом диапазоне веществ типа $\mathrm{NaCl}[10]$. При реализации DAC-метода обычно лепесток металлического образца помещается в прокладки (рений и т.п.), чтобы избежать химического взаимодействия образца с алмазом и уменьшить темп нагрева последнего. Опыты, как правило, проводятся при давлениях выше $10 \mathrm{GPa}$, чтобы избежать возможной графитизации алмаза при более низких давлениях. Известно, что для титана пленка кислорода, которая обычно образуется на поверхности $\alpha$-фазы, переходит в объем после $\alpha-\beta$-перехода. Этот вопрос и проблема чистоты атмосферы в рассматриваемых опытах также не обсуждается. При экстраполяции опытных $T_{m}(p)$ данных работы [8] на низкие давления для Мо и Ті можно получить низкие значения для начального наклона лини плавления $\left(d T_{m} / d p\right)_{p \rightarrow 0}=7-10 \mathrm{~K} \cdot \mathrm{GPa}^{-1}$. Это противоречит традиционным оценкам этой величины для ряда других тугоплавких металлов [11] и, вообще говоря, ставит под сомнение достоверность рассматриваемых опытов. Относительно молибдена, его линии плавления и производной $\left(d T_{m} / d p\right)_{p \rightarrow 0}$ имеется обширная литература, и современная оценка составляет $35-40 \mathrm{~K} \cdot \mathrm{GPa}^{-1}$ [12]. В обзоре 2006 г. [13] Еррандонеа повторяет рисунок с опытными данными на линии плавления титана, приводит новые примеры расхождения опытных данных на линии плавления элементов, полученных DAC-методом, и подчеркивает необходимость в проведении дальнейших исследований.

Для титана результаты новых измерений при давлениях до $118 \mathrm{GPa}$ и температурах до $2850 \mathrm{~K}$ были опубликованы только в 2015 г. [14]. Авторы использовали тот же DAC-метод с лазерным нагревом образца. Процесс плавления дополнительно фиксировался с помощью гамма-спектрометра по потере кристалличности образца. По мнению авторов этой работы, ранее в [8] при проведении измерений для титана могли наблюдать не плавление, а процесс рекристаллизации неотожженного образца при температуре ниже температуры плавления.
Влияние микроструктуры, состояния поверхности, чистоты исходных образцов металла на результаты измерений процессов при ударном сжатии титана отмечается также в работе [15]. В работе [14] приводятся 7 опытных точек $T_{m}(p)$ в интервале давлений 47-118 GPa. Одновременно в этой работе приведены для титана результаты $a b$ initio молекулярно-динамических расчетов и затем в разных приближениях (однофазная и двухфазная модели плавления) оценки линии плавления. Результаты расчетов по двухфазной модели (твердая-жидкая фазы) близки к опытным данным и представлены в работе уравнением Симона:

$$
T_{m}=T_{m}^{0}\left(1+p / p^{*}\right)^{1 / c},
$$

где $T_{m}^{0}=1941 \mathrm{~K}, p^{*}=7.0 \mathrm{GPa}, c=7.19$. Для многих металлов ПС коэффициент $c=1.5$ [3]. В то же время, например в работе [16], при обобщении данных на линии плавления никеля этот параметр оказался равным $c=4.5$. При рассмотрении широкого спектра значений $c$ для уравнения Симона надо иметь в виду, что само уравнение носит эмпирический характер. Этой форме уравнения соответствует следующая связь с термодинамическими свойствами на линии плавления: $c\left(p+p^{*}\right)=$ $=T_{m} \Delta S / \Delta V$. При нормальных условиях производная $\left(d T_{m} / d p\right)_{p \rightarrow 0}=T_{m}^{0} /\left(c p^{*}\right) \quad$ и для приведенных выше параметров из работы [14] для титана она равна $38.6 \mathrm{~K} \cdot \mathrm{GPa}^{-1}$ и согласуется с оценкой этой величины для ряда других $d$-металлов [11]. С другой стороны, проведенная нами аппроксимация опытных $T_{m}(p)$ данных для титана [14] в рамках метода наименьших квадратов (МНК) говорит о том, что коэффициент корреляции $c, p^{*}$ параметров $r \rightarrow 1$, и нужна дополнительная информация для уточнения параметров и анализа согласованности данных для титана при низком и высоком давлении.

Для титана имеется ряд публикаций, в которых приводятся результаты расчетов термодинамических свойств различных твердых фаз и жидкости, а также данные о фазовом равновесии между ними в широком интервале давлений и температур. В работах $[17,18]$ расчеты проведены на базе широкодиапазонных УС, в работах $[19,20]$ - с помощью метода молекулярной динамики (МД) (модели погруженного атома). Оценки производной в $[17,19,20]\left(d T_{m} / d p\right)_{p \rightarrow 0} \approx 50 \mathrm{~K} \cdot \mathrm{GPa}^{-1}$. В работе 2005 г. [18], опубликованной до появления опытных данных [14], производная $\left(d T_{m} / d p\right)_{p \rightarrow 0} \approx 7 \mathrm{~K} \cdot \mathrm{GPa}^{-1}$, и на рисунке показана тройная точка равновесия двух твердых $\beta, \omega$ и жидкой фаз при давлении $\sim 40 \mathrm{GPa}$.

Можно отметить, что приведенные выше оценки производной $\left(d T_{m} / d p\right)_{p \rightarrow 0}$ в основном значительно выше, чем в экспериментальной работе 2001 г. [8]. Для уточнения этих оценок естественно обратиться к расчету производной по уравнению Клапейрона-Клаузиуса и проанализировать опытные данные по скачкам объема $\Delta V_{m}$ и энтропии плавления $\Delta S_{m}$ титана при атмосферном давлении. 


\section{Атмосферное давление}

Что касается величины $\Delta S_{m}=\Delta H_{m} / T_{m}$, то в свое время при работе над монографией [4] была проведена совместная МНК-аппроксимация опытных данных по декрементам энтальпии и теплоемкости $\alpha$-, $\beta$-фаз и жидкого титана в интервале температур $300-2430 \mathrm{~K}$ и получена оценка $\Delta H_{m}=14.39 \pm 0.9(3 \sigma) \mathrm{kJ} \cdot \mathrm{mol}^{-1}$ с относительной погрешностью $\delta \Delta H_{m} \approx 6 \%$. С этой величиной практически совпадает оценка $\Delta H_{m}=14.15 \mathrm{~kJ} \cdot \mathrm{mol}^{-1}$ из широко распространенного обзора [21].

Для титана прямые измерения величины скачка объема $\Delta V_{m}$ отсутствуют. Это связано, в частности, с высокой химической активностью особенно жидкого титана, что затрудняет выбор материала контейнера для проведения опытов в стационарных условиях [22]. В работах 80-х годов прошлого века по импульсному нагреву проводника током, например, в работе [23] для титана изменение объема при плавлении показано лишь на графике в небольшом масштабе, откуда трудно выделить величину скачка и оценить его неопределенность. На пути оценки величины скачка, т. е. разницы объемов сосуществующих фаз $\Delta V_{m}=V_{l}\left(T_{m}^{0}\right)-V_{s}\left(T_{m}^{0}\right)$, возникает проблема оценки плотности $d\left(T_{m}^{0}\right)=1 / V\left(T_{m}^{0}\right)$ и ее неопределенности, как можно будет видеть, в первую очередь, для твердой фазы при температуре плавления.

Для анализа согласования опытных $T_{m}(p)$ данных титана при высоких давлениях с величиной производной $\left(d T_{m} / d p\right)_{p \rightarrow 0}$ желательно для последней располагать оценкой неопределенности $\delta\left(\left(d T_{m} / d p\right)_{p \rightarrow 0}\right)<25-30 \%$ и для величины скачка объема, имея в виду приведенную выше погрешность скачка энтропии, оценкой $\delta\left(\Delta V_{m}\right)<20-25 \%$. Имеющиеся в литературе данные о плотности жидкого титана при нормальной температуре плавления, например, $d_{l}\left(T_{m}^{0}\right)=4.13 \mathrm{~g} \cdot \mathrm{cm}^{-3}$ [4] и оценки для скачка объема $\Delta V_{m} \approx 2.5 \%$ позволяют сделать грубую оценку погрешности $\delta\left(\Delta V_{m}\right)$. Если сумма погрешностей значений плотности твердой и жидкой фаз будет равна $0.04 \mathrm{~g} \cdot \mathrm{cm}^{-3}$, т.е. в сумме $1 \%$, то мы получим оценку $\delta\left(\Delta V_{m}\right) \approx 40 \%$. Что касается плотности жидкого титана, то в настоящее время известны результаты трех независимых измерений $d_{l}\left(T_{m}^{0}\right), \mathrm{g} \cdot \mathrm{cm}^{-3}$ : 4.14 ([24] метод электромагнитной левитации капли), 4.13 ([25] метод висящей капли, электронно-лучевой нагрев образца), 4.17 ([26] метод электростатической левитации капли), среднее из которых с учетом погрешности отдельных данных составляет $4.14 \pm 0.02 \mathrm{~g} \cdot \mathrm{cm}^{-3}$. Надо отметить, что авторами работ $[25,26]$ разными методами проведены измерения плотности $d_{l}\left(T_{m}^{0}\right)$ практически всех тугоплавких металлов в интервале температур 1500-3700 K. Расхождение этих опытных данных не превышает $1 \%$, что является свидетельством высокого уровня современных теплофизических исследований.

Для высокотемпературной ОЦК $\beta$-фазы титана известны лишь две работы, в которых приводятся опытные данные по линейному коэффициенту теплового расширения (ЛКТР) до температуры $1600-1650 \mathrm{~K}[27,28]$.

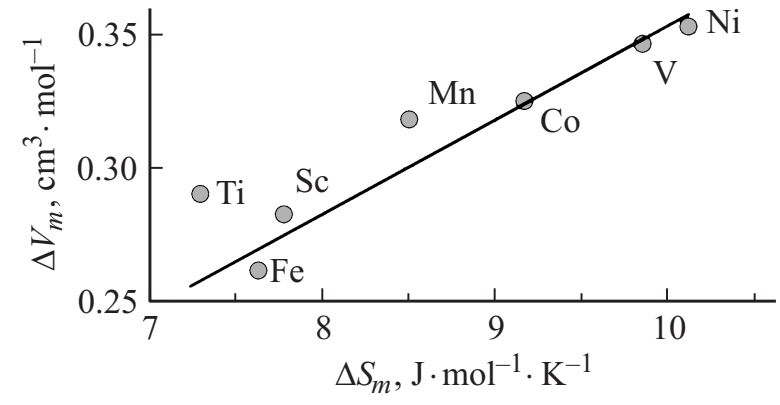

Рис. 1. Корреляция данных $\Delta V_{m}=f\left(\Delta S_{m}\right)$. В масштабе графика $K=\Delta V_{m} \cdot 1000 / \Delta S_{m}$.

Высокая химическая активность и заметная текучесть образца не позволили провести дилатометрические измерения линейного расширения при более высоких температурах. Оценка плотности твердого титана $d_{s}\left(T_{m}^{0}\right)=4.26 \mathrm{~g} \cdot \mathrm{cm}^{-3}$ была получена при экстраполяции ЛКТР на $300 \mathrm{~K}$ до температуры плавления [4]. Погрешность этого значения плотности, имея в виду все особенности поведения твердой фазы при подходе к $T_{m}^{0}$, трудно оценить менее $1.5 \%$. При этом оценка погрешности скачка объема $\delta\left(\Delta V_{m}\right) \approx 80 \%$, что делает бесперспективным использование рассматриваемых данных о скачке объема $\Delta V_{m}$ для анализа согласованности данных на линии плавления при высоких и низких давлениях.

В этой ситуации мы рассмотрели возможности другого метода оценки величины $\Delta V_{m}$, основанного на анализе зависимости $\Delta V_{m}=f\left(\Delta S_{m}\right)$ для $3 d$-элементов ПС. В качестве информации о скачках энтропии были использованы значения в основном из обзора [21]. В качестве „опытных“ данных для скачков объема были приняты значения $\Delta V_{m}=\delta\left(\Delta V_{m}\right) V_{l}\left(T_{m}^{0}\right)$, где $\delta\left(\Delta V_{m}\right)-$ оценки относительных погрешностей скачков объема из обзора Станкуса [22], значения $V_{l}\left(T_{m}^{0}\right)$ - опытные данные [25]. Из обработки для $3 d$-элементов были исключены данные для хрома по двум причинам: 1) электронная оболочка атома $\mathrm{Cr} \ldots 3 d^{5} s^{1}$ отличается от структуры $\ldots .3 d^{n} s^{2}$ других $3 d$-элементов, 2) физикохимические свойства в твердой фазе имеют особенности по сравнению со свойствами других элементов этого периода [29]. В 2015 г. были опубликованы результаты новых измерений плотности твердого и жидкого никеля, полученные в Институте теплофизики СО РАН [30] с использованием метода дилатометра для твердой фазы и метода гамма-излучения для обеих фаз. Рекомендуемое в этой работе значение скачка объема и оценка его погрешности $\Delta V_{m}=(4.07 \pm 0.15) \%$ использованы нами в дальнейшей обработке. Как показал анализ, рассматриваемый массив семи „опытных“ точек можно описать с помощью простой зависимости $\Delta V_{m}=K \Delta S_{m}$, где параметр $K$ и его статистическая оценка погрешности $3 \sigma$ равны $K=35.3 \pm 1.2 \mathrm{~K} \cdot \mathrm{GPa}^{-1}$. Параметры более сложных моделей на этом массиве данных оказываются 
незначимыми. Результаты аппроксимации показаны на рис. 1. Разброс точек в первом приближении носит случайный характер. Если ориентироваться не на статистическую оценку погрешности модели 3\%, а на максимальный разброс точек, то оценка погрешности $\delta K=10 \%$, и именно эту оценку мы используем далее при согласовании данных. Для титана расчетные значения $\Delta V_{m}=0.257 \pm 0.026 \mathrm{~cm}^{3} \cdot \mathrm{mol}^{-1}$ и плотность твердой фазы $d_{s}\left(T_{m}^{0}\right)=4.22 \pm 0.03 \mathrm{~g} \cdot \mathrm{cm}^{-3}$.

\section{Проблема тройной точки $\beta-\omega$-жидкая фаза}

Далее мы рассматриваем линию плавления $T_{m}(p)$ $\beta$-фазы титана в интервале от атмосферного давления до 90-100 GРа. При этом надо иметь в виду следующее обстоятельство. У металлов, которые в твердой фазе имеют разные полиморфные формы, линия равновесия последних может пересекать линию плавления и при этом возникает тройная точка двух твердых фаз и жидкости. Классическим, хорошо изученным примером такой ситуации является тройная точка равновесия ГЦК-ОЦК-жидкая фазы железа при давлении $5.2 \mathrm{GPa}$ и температуре $1990 \mathrm{~K}$ [5]. В твердом титане кроме полиморфных $\alpha$ - и $\beta$-модификаций существует гексагональная $\omega$-фаза [7]. После тройной точки $\alpha-\beta-\omega$-фаз $(8 \mathrm{GPa}, 910 \mathrm{~K})$ линия равновесия $\beta-\omega$-фаз в $T_{m}-p$-координатах идет вверх. Ф. Банди в 1963 г. провел измерения на линии равновесия этих фаз в интервале давлений 8-12 GPa и определил величину наклона $d T_{\beta \omega} / d p \approx 20 \mathrm{~K} \cdot \mathrm{GPa}^{-1}$ [31]. Естественно задать вопрос: может ли линия равновесия $\beta-\omega$-фаз с ростом давления пересечь линию плавления $\beta$-фазы, после чего надо будет рассматривать плавление $\omega$-фазы. В 2012 г. опубликованы результаты новых измерений плотности Ti, Zr, Hf, полученные с помощью метода алмазных наковален при температурах до $2200 \mathrm{~K}$ и давлениях до $35 \mathrm{GPa}$ [32]. Используя расчетные CALPHADпроцедуры, автор построил УС твердых фаз этих металлов, включая линии равновесия фаз. Для титана линия равновесия $\beta-\omega$-фаз построена от тройной точки до $35 \mathrm{GPa}$ и $1800 \mathrm{~K}$. При высоких давлениях значение наклона этой линии равно $d T_{\beta \omega} / d p \approx 40 \mathrm{~K} \cdot \mathrm{GPa}^{-1}$. При ее экстраполяции вверх до $60 \mathrm{GPa}$ можно видеть, что линия $\beta-\omega$-равновесий может пересечь линию плавления $T_{m}(p)$ данных из работы [14]. В то же время авторы работы [14] предварительно провели с помощью DACметода детальные исследования свойств и равновесий $\alpha-\beta-\omega$-фаз в широком интервале параметров [33], в частности, измерения параметра решетки ОЦК $\beta$-фазы в интервале давлений 35-75 GPa. Это позволяет считать, что в работе [14] получены опытные данные именно для плавления $\beta$-фазы. Тем самым мы можем предположить, что если линия $\beta-\omega$-равновесий и пересекает линию плавления, то это может происходить при давлениях выше 90-100 GPa.

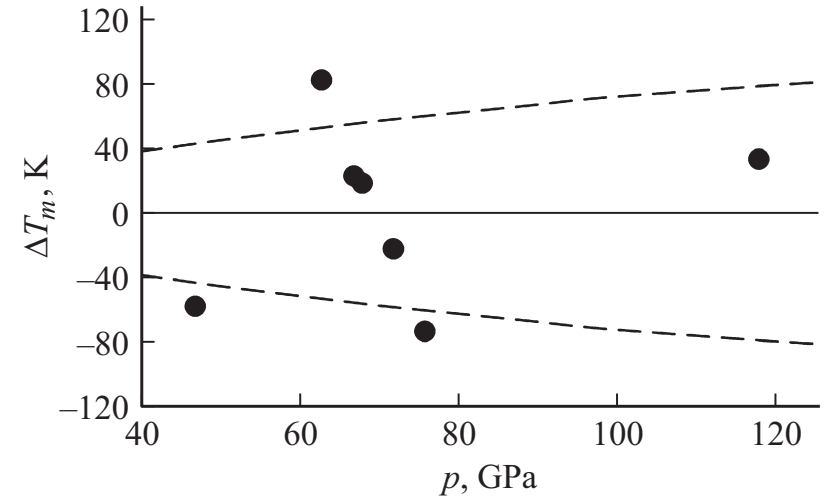

Рис. 2. Результаты аппроксимации данных.

\section{Результаты согласования данных}

Следуя логике работы 2015г. [14], мы провели совместную обработку данных на линии плавления титана, используя информацию об опытных $T_{m}(p)$ данных из этой работы и нашу оценку производной $\left(d T_{m} / d p\right)_{p \rightarrow 0}$. В качестве модели линии плавления принято трехпараметрическое уравнение Симона (2), где $c$ и $p^{*}$ - варьируемые параметры. Совместная обработка опытных данных (7 точек $T_{m}(p)$ с погрешностью $\Delta T_{m}=200 \mathrm{~K}$ из работы [14]) и значения наклона с погрешностью $\Delta\left(\left(d T_{m} / d p\right)_{p \rightarrow 0}\right)=4 \mathrm{~K} \cdot \mathrm{GPa}^{-1}$ проведена в рамках весового нелинейного МНК. В качестве априорной информации использована оценка параметра $c=7 \pm 3$. МНК функционал $S=0.43$, отклонение опытных данных от расчетной зависимости носит случайный характер и не превышает принятых погрешностей. Параметры $p^{*}=7.81 \pm 1.62 \mathrm{GPa}$, $c=7.04 \pm 0.88$, коэффициент корреляции ошибок параметров, выраженный через элементы матрицы ошибок МНК, $r=\operatorname{cov}\left(c p^{*}\right) /\left(D(c) D\left(p^{*}\right)\right)^{0.5}=0.89$. Если в обработке использовать только опытные $T_{m}(p)$ данные, коэффициент $r \rightarrow 1$. Для оценки доверительных погрешностей расчетных значений $\Delta_{p} Y$ при доверительной вероятности $P$ использовано в первом приближении стандартное выражение правила переноса случайных ошибок [34]. Принято $P \approx 0.9$, коэффициент согласия (coveradge factor) $t_{p}=5$ с учетом допущений для использованного варианта МНК (независимые оценки погрешностей опытных данных, бедная статистика и т.п.). Разброс опытных данных $T_{m}(p)$ относительно сглаживающей зависимости и коридор погрешностей $\Delta_{p} Y$ в интервале давлений до $90 \mathrm{GPa}$ показаны на рис. 2.

\section{Заключение}

1. Проведено согласование выбранных на основе критического анализа данных на линии плавления титана при низком и высоком давлении до $90 \mathrm{GPa}$. Остается открытым вопрос о возможности появления тройной точки 
$\beta$-, $\omega$ - и жидкой фаз на лини плавления при давлении $>100 \mathrm{GPa}$. Отсутствие опытных данных о плотности твердого титана от $1600 \mathrm{~K}$ до температуры плавления и прямых измерений скачка объема затрудняет всю процедуру согласования.

2. Продемонстрирована и использована в дальнейшей обработке линейная зависимость величин скачков объема и энтропии при плавлении $d$-элементов $\mathrm{Sc}, \mathrm{Ti}, \mathrm{V}, \mathrm{Co}$, $\mathrm{Mn}, \mathrm{Fe}, \mathrm{Ni}$ при нормальном давлении.

3. Приведенные выше оценки доверительных погрешностей величин на линии плавления основаны на вероятностных представлениях, и для оценки надежности полученных результатов нужны дополнительные исследования на линии плавления титана.

4. Для анализа причин расхождения результатов определения $T_{m}(p)$ данных, полученных методом алмазных наковален в работах разных групп авторов, необходимы дополнительные исследования на метрологическом уровне факторов, влияющих на достоверность результатов.

5. Работа может рассматриваться как дополнительная глава монографии по теплофизическим свойствам титана и его сплавов [4].

Статья подготовлена при поддержке гранта РФФИ 1708-00736.

\section{Список литературы}

[1] Бушман А.В., Фортов В.Е. // УФН. 1983. Т. 140. Вып. 2. C. $177-232$.

[2] Белащенко Д.К. // УФН. 2013. Т. 183. Вып. 12. С. 12811322.

[3] Кулямина Е.Ю., Зищерман В.Ю., Фокин Л.Р. // ЖТФ. 2017. T. 87. Вып. 1. С. 59-64.

[4] Chekhovskoi V.Ya., Fokin L.R., Peletskii V.E., Petukhov V.A., Shur B.A. Handbook of titanium-based materials: Thermophysical properties, data and studies. NY: Begell house, 2007. $275 \mathrm{p}$.

[5] Тонков Е.Ю. Фазовые диаграммы элементов при высоких давлениях М.: Физматлит, 1979. 192 с.

[6] Yang D.A. Phase diagrams of elements. L.: Univ. California Press, 1991. 284 p.

[7] Tonkov E.Yu., Ponytovsky E.G. Phase transformation of elements under high pressure. Roca Raton: CRC press, 2007. $380 \mathrm{p}$.

[8] Errandonea D., Schwager B., Ditz R. et al.// Phys. Rev. 2001. Vol. 63. P. 132104(13).

[9] Shen G., Prakapenka V., Rivers M., Sutton S. // Phys. Rev. Lett. 2004. Vol. 92. P. 185701.

[10] Vereshagin L.F., Fateeva N.S. // High Temp.-High Press. 1977. Vol. 9. P. 619-628.

[11] Фокин Л.Р. // Мониторинг. Наука и технологии. 2011. № 4. C. 103-107.

[12] Hrubiak R., Meng Y., Shen G. // Nature Commun. 2017. Vol. $8: 14562$

[13] Errandonea D. // J. Phys. Chem. Sol. 2006. Vol. 67. P. 20182037.

[14] Stutzmann V., Dewaele A., Bouchet J. et al. // Phys. Rev. B. 2015. Vol. 92. P. 224110(10).
[15] Трунин Р.Ф., Симаков Г.В., Медведев А.В. // ТВТ. 1999. Т. 37. Вып. 6. С. 881-886.

[16] Errandonea D. // Phys. Rev. B 2013. Vol. 87. P. 054108(5).

[17] Kerley $G$. Equation of state for titanium and Ti6Al14V alloy. Rt. SAND 2003-3758. Sandia Nat. Lab. Albuquerque, USA, 2003. 28 p.

[18] Pecker S., Eliezer S., Fisher D., Hines Z. // J. Appl. Phys. 2005. Vol. 98. P. 043516(12)

[19] Kim Y.-M., Lee B.-J., Baskes M.I. // Phys. Rev. 2006. Vol. 74. P. 014101(12).

[20] Zeng Z.-Y., Cai L.-C., Chen X.-R., Jing F.-Q. // J. Appl. Phys. 2011. Vol. 109. P. 043503(7).

[21] Dinsdale A.T. // CALPHAD. 1991. Vol. 15. N 4. P. 317-425.

[22] Станкус C.В. Измерение плотности элементов при плавлении. Методы и экспериментальные данные. Новосибирск: Институт теплофизики СО РАН. Препринт № 24791. $79 \mathrm{c}$.

[23] Gathers G.R. // Int. J. Thermophys. 1983. Vol. 4. N 3. P. $271-$ 278.

[24] Sato T., Shiraishi Y., Sakuma Y. // Trans. Iron and Steel Inst of Japan. 1969. Vol. 9. P. 118-126.

[25] Физическая химия неорганических материалов. Т. 2: Поверхностное натяжение и термодинамика металлических расплавов / Еременко В.Н., Иванов М.И., Лукашенко Г.М. и др. Киев: Наукова думка, 1988. С. 59-103.

[26] Ishikawa T., Paradis P.-F. // Adv. Mater. Res. 2009. Vol. 11. P. 173-195.

[27] Williams D.N. // Trans. Met. Soc. AIME. 1961. Vol. 221. N 2. P. 411-412.

[28] Schmitz-Pranghe N., Dunner P. // Z. Metallkunde. 1968. Vol. 58. N 5. P. 377-382.

[29] Лякишев Н.П., Гасик М.И. Металлургия хрома. М.: ЭЛИЗ, 1999. $581 \mathrm{c}$.

[30] Abdullaev R.N., Kozlovski Yu.M., Khairulin R.A., Stankus S.V. // Int. J. Thermophys. 2015. Vol. 36. N 4. P. 603-619.

[31] Bundy F.P. GE Research Lab. Rep. N 63-RL-3184C. USA, October, 1963.

[32] Hrubiak R. Exploring thermal and mechanical properties selected transition elements under extreme conditions: experiments at high pressures and high temperatures. Thesis: Florida Int. Univ. USA. 2012.

[33] Dawaele A., Stutzmann V., Fouchet J. et al. // Phys. Rev. B. 2015. Vol. 91. P. 134108(13).

[34] Фокин Л.Р., Калашников А.Н. // ИФЖ. 2016. Т. 89. Вып. 1. C. 240-249. 